\title{
Fellowship training in Acute Care Surgery: from inception to current state
}

\author{
Kimberly A Davis, ${ }^{1}$ Gregory J Jurkovich ${ }^{2}$
}

${ }^{1}$ Department of Surgery, Yale School of Medicine, New Haven, Connecticut, USA ${ }^{2}$ Department of Surgery, UC Davis Health System, Sacramento, California, USA

\section{Correspondence to} Dr Kimberly A Davis; kimberly. davis@yale.edue000004

Received 4 April 2016 Revised 5 May 2016 Accepted 9 May 2016
To cite: Davis KA, Jurkovich GJ. Trauma Surg Acute Care Open Published Online First: [please include Day Month Year] doi:10.1136/tsaco-2016000004

\begin{abstract}
Recognizing the need for urgent and emergent surgical care across America, the American Association for the Surgery of Trauma developed and implemented, and oversees, the Acute Care Surgery Fellowship Training Program. Now in its 10th year, the fellowship has become an established post-General Surgery Fellowship Training Program, with 20 approved programs and 82 fellows trained. Consistent with the desire to have this non-Accreditation Council for Graduate Medical Education (ACGME) fellowship one with the highest standards, several educational improvements have occurred since its origin. The following is an account of the background and evolution of what has become a significant educational contribution to surgery.
\end{abstract}

\section{RATIONALE FOR DEVELOPMENT OF THE ACUTE CARE SURGERY (ACS) FELLOWSHIP}

As with many events in medicine, the evolution and development of ACS was born out of necessity and innovation. By the end of the 20th century, it was recognized that there was an insufficient number of physicians willing to participate in emergency call panels, a problem highlighted in the Institute of Medicine's report entitled 'Hospital Based Emergency Care at the Breaking Point'. ${ }^{1}{ }^{2}$

Compounding this crisis in access to emergency care, is continued and worsening workforce shortage of general surgeons, and aging of the current surgical workforce. ${ }^{2}{ }^{3}$ Although the workforce in non-surgical specialties has grown steadily over time, the number of surgeons trained has remained stable for $>20$ years (figure 1). Between 1981 and 2006, the US population grew $31 \%$, while the number of general surgeons grew by $4 \%{ }^{4}$ The American Association of Medical Colleges estimates that a $35 \%$ increase in the number of surgeons will be necessary to meet clinical demands by 2025 (figure 2). Just as the needs of the injured patient drove the development of the field of trauma surgery, so did the needs of the emergency general surgery patient drive the development of the ACS paradigm. ${ }^{5}$ This specialty enhances the training of young surgeons in the areas of trauma, surgical critical care, and time-sensitive general surgery.

Although often used interchangeably, 'emergency general surgery' and 'acute care surgery' have different meanings. Whereas emergency general surgery refers to acute general surgical disorders, acute care surgery includes surgical critical care and the surgical management of acutely ill patients with a variety of conditions including trauma, burns, surgical critical care or an acute general surgical condition. The challenges in caring for these patients include around-the-clock readiness for the provision of comprehensive care, the often constrained time for preoperative optimization of the patient, and the greater potential for intraoperative and postoperative complications due to the emergent nature of care.

\section{THE HISTORY OF ACS FELLOWSHIP TRAINING}

In 2005, the American Association for the Surgery of Trauma (AAST) responded to the crisis in access to emergency surgeons by creating the foundations for the training of surgeons in ACS. The AAST conceptualized a training program that emphasized trauma surgery, surgical critical care, and focused expertise in emergency general surgery conditions. The training was designed to create a versatile surgeon able to confront a host of acute surgical disease processes. A curriculum was developed that included a focus on clinical experience but also defined the operative expectations of a trainee in an accredited program. An initial list of 'essential and desirable' cases was created, which focused on a broad range of predominantly trauma case types divided into anatomic region. The initial curriculum dictated mandatory components while also allowing some flexibility for the fellows in terms of rotations. With some creativity, programs could capitalize on not only the individual's strengths but address their weaknesses as well. Rotations in thoracic surgery, transplant/hepatobiliary/pancreatic, and vascular were encouraged to expand operative experience in these anatomically and physiologically challenging areas, but were not mandated in the initial curriculum. ${ }^{6}$ The goal of the ACS Fellowship was to master complex operative procedures in trauma and emergency general surgery, in conjunction with an Accreditation Council for Graduate Medical Education (ACGME)-approved Surgical Critical Care Fellowship.

The process for program approval was structured in a manner similar to that of a trauma center verification visit. Programs interested in applying to become an approved ACS program were required to submit previsit data including a Program Information Form (PIF). Surgeons from the AAST ACS Committee perform the verification visits, meeting with program and institutional leadership, faculty, and trainees at the resident and fellow level. The visit assesses the volume of cases available and the educational structure as well as any potential impact on general surgery residency training. Initial approval was granted for a period no $>4$ years, with planned revisits to maintain status as an approved AAST ACS program. In 2008, the first ACS Fellowship was approved by the AAST Board of Managers, and training started. Since 2008, a total of 20 programs have been approved by the 


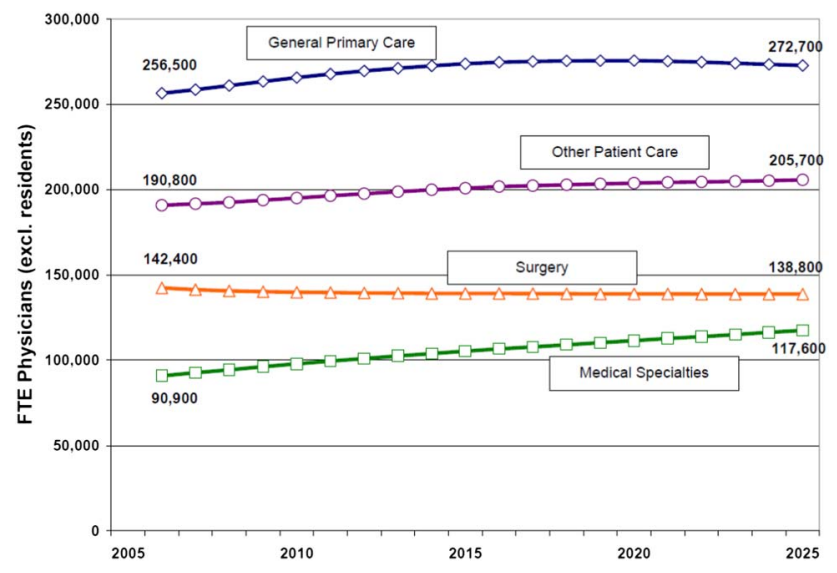

Figure 1 Projections of Physicians by Specialty Group. FTE, full time employee. Source: Dill et al, ${ }^{23}$ used with permission.

AAST. As of June of 2015, 82 fellows have been trained.

In 2009, the AAST launched the first iteration of a written examination for successful completion of the fellowship. This examination was designed to address whether the fellowships were meeting appropriate educational metrics. As with most examinations, the early versions required substantial revision, a process that continues on an annual basis as the AAST continues to refine the fellowship. In 2015, an in-training examination was added, to better demonstrate improvements in fellow knowledge over the period of the ACS Fellowship year (box 1).

In 2010, the AAST launched a case log system (Infotech, San Diego, California, USA) to track the operative experience of the fellows. Four years of data are available for review. The average number of operative procedures per fellow ranges from 300 to 350 , with $\sim 50 \%$ of the cases having more than one captured current procedural terminology (CPT) code. In 2014, a review of the fellowship case logs demonstrated a high degree of institutional and individual variability in the case mix, with $\sim 50 \%$ of fellows not meeting the case types specified in the initial curriculum. ${ }^{6}$ Most notably, ACS trainees lacked adequate exposure to head/neck surgery, thoracic surgery, and vascular surgery as defined by the ACS curriculum.

\section{TRAJECTORY OF THE ACS FELLOWSHIPS}

In light of concerns regarding case volume and mix, the operative curriculum for the ACS Fellowship was restructured during

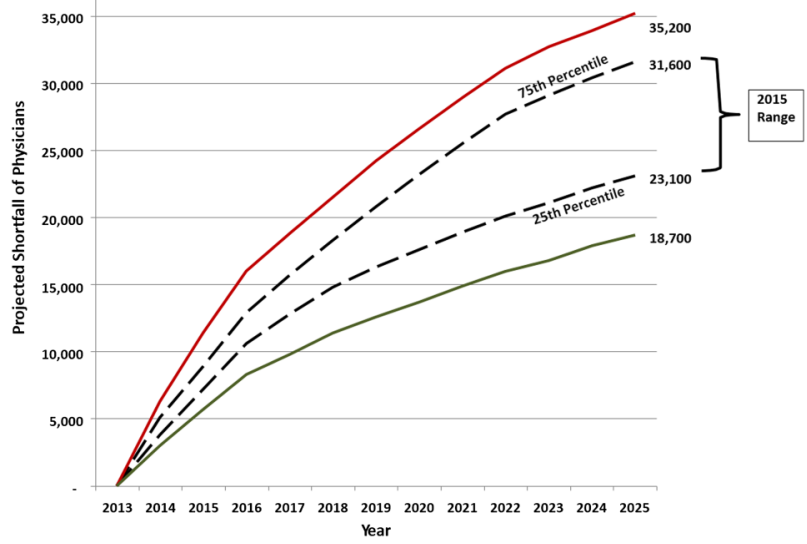

Figure 2 Projected Surgical Physician Shortfall, 2013-2025. Source: IHS Inc, ${ }^{4}$ used with permission.
Box 1 Approved American Association for the Surgery of Trauma (AAST) Acute Care Surgery (ACS) Fellowship Training Programs

- University of California, San Francisco-Fresno, Fresno, California

- University of Colorado School of Medicine, Denver, Colorado

- University of Maryland/R Adams Cowley Shock Trauma Center, Baltimore, Maryland

- University of Nevada School of Medicine, Las Vegas, Nevada

- University of Pittsburgh Medical Center, Pittsburgh, Pennsylvania

- Massachusetts General Hospital, Boston, Massachusetts

- University of Texas Health Science Center, Houston, Texas

- Vanderbilt University, Nashville, Tennessee

- UMDNJ-Robert Wood Johnson Medical School, New Brunswick, New Jersey

- Wake Forest Baptist Medical Center, Winston-Salem, North Carolina

- East Carolina University/Vidant Medical Center, Greenville, North Carolina

- University of Arizona, Tucson, Arizona

- Baystate Medical Center, Springfield, Massachusetts

- Hartford Hospital/University of Connecticut, Hartford, Connecticut

- Wright State University, Dayton, Ohio

- Yale University, New Haven, Connecticut

- Orlando Regional Medical Center, Orlando, Florida

- University of Florida-Gainesville, Gainesville, Florida

- Indiana University Medical School, Indianapolis, Indiana

- Emory University Medical School, Atlanta, Georgia

Source: Ref. ${ }^{24}$

2014. Previously elective rotations in thoracic surgery and vascular surgery became required rotations. Curricular changes included the identification of a minimum number of operative cases needed in specific body regions, similar to defined case volumes in general surgery. The desired case volume provides guidance to the fellows, program directors and subspecialty colleagues as to the types of cases deemed important for the fellows' training. ${ }^{7}$ In an effort to distinguish the surgical training in the fellowship from that obtained in the general surgical residency, metrics regarding patient comorbidity as defined by the AAST emergency general surgery disease grading scales ${ }^{8}$ and the technical difficulty of the operative case were added to the case $\log$ system. In 2015, these changes were implemented as requirements for successful completion of the fellowship.

The AAST and ACS Fellowships will continue to build on the strong foundation of process and structure that already exists. In 2015, a series of educational modules was created, addressing topics in thoracic and vascular surgery deemed important to the ACS fellow. Each module has several maintenance of certification (MOC)-type questions that will be added to the question bank for the end of fellowship examination. These modules support the alignment of the fellow education with testing at the end of fellowship, further closing perceived gaps. In addition to more in-depth education at a level beyond that expected during residency, the modules offer advanced techniques for complex operative procedures on patients who, due to severity of illness, do not have the luxury of preoperative optimization. 
In keeping with the structure of general surgery training programs, an in-service examination has been developed to assess the baseline knowledge of fellows at the beginning of their ACS year. Information regarding performance, including topics missed by the fellows on the examination, are given to the program directors, to allow the program directors to direct additional education to areas of weakness identified in the examination process. The end of training examination continues to undergo revision, most recently de-emphasizing critical care questions, as all ACS fellows will be eligible to take the American Board of Surgery (ABS) examination in Surgical Critical Care.

The goals of training ACS surgeons are to demonstrate mastery and future leadership in the field of ACS, above and beyond that learned in a general surgery residency. Certification in ACS is currently offered through the AAST. Unlike other specialty training paradigms, which tend to focus on a specific organ or operative technique, this paradigm strives to create a broad-based surgical specialist, specifically trained in the treatment of acute surgical disease across a wide array of anatomic regions. $^{7}$

All fellowship programs are required to participate in the match process through the National Residency Match Program (NRMP). Ongoing compliance with match participation is monitored at the time of program re-verification. In addition, central monitoring is now possible through a centralized application process (Surgical Critical Care and Acute Care Surgery Fellowship Application Service (SAFAS)) created in conjunction with the Society of Surgical Critical Care Program Directors. In the most recent match year, all programs were in compliance with match participation. To date, 12 of the 20 ACS Fellowship programs have undergone successful reverification site visits.

\section{IMPACT OF ACS FELLOWSHIPS}

As with the addition of any surgical fellowship, there is always a concern for any adverse impact that the fellowship may have on the general surgery residents' training. One study sought to determine how the addition of an ACS fellow impacted the training of the current general surgery residents by comparing general surgery ACGME case log data before and after the initiation of the AAST approved ACS Fellowship. The study found that there was no significant change in the number of cases performed by the chief residents. Furthermore, general surgery residents believed that the ACS fellow added value especially as an educator. $^{9}$

Additional studies have focused mainly on resident and surgeon interest, and satisfaction with ACS. In 1992, Richardson and Miller ${ }^{10}$ surveyed nearly 1800 surgery residents, and found only $18 \%$ wanted any part of trauma care in their career plans. Interest in trauma-only fellowships was very low, and disaffection with trauma care was predominant in the survey. Primary reasons for these negative feelings were the large amount of non-operative care rendered in treating blunt trauma patients, the unsavory type of patients encountered with most penetrating trauma injuries, and lifestyle issues. Complaints about the structure of trauma rotations in many institutions and the poor role-modeling of trauma surgeons were cited as reasons for respondents not pursuing trauma care as a career interest. ${ }^{10} 11$

Recruitment into the field of trauma and critical care surgery was traditionally poor, as demonstrated by $\sim 18 \%$ of fellowship positions unfilled in 2011. In 2015, the number of filled fellowship positions had improved and only $10 \%$ of positions were unfilled. ${ }^{12}$ The number of Surgical Critical Care Fellowship positions increased by $34 \%$ over the 5 -year period, while the number of applicants increased by 61\% (figure 3). In 2013, Coleman $e t \mathrm{al}^{13}$ surveyed residents regarding a career path in ACS that yielded a greater interest and understanding of ACS as a career. Overall, these studies showed a much greater interest and understanding of ACS as a career choice, encompassing surgical critical care and emergency general surgery.

Surgeon satisfaction is improved with an ACS model compared with more traditional call schedules. On average, surgeons in institutions with an ACS model have higher satisfaction scores than those surgeons not using an ACS model. ${ }^{14}$ ACS surgeons and non-ACS surgeons both reported improved job satisfaction with the implementation of an ACS service. ${ }^{15}$ As the fellowship matures and acute care surgeons enter the workforce, this will hopefully become a more attractive option for rising surgical residents.

Several studies have focused on surgeon productivity since the implementation of an ACS model. One study compared operative productivity before and after the implementation of ACS, and demonstrated a $66 \%$ increase in operative volume with an ACS division in place. Similarly, there was an increase in Evaluation and Management (E\&M) work Relative Value Unit (wRVU) production as well as a rise in procedural wRVU production for both ACS and non-trauma surgeons. ${ }^{15}$ Additional studies have also shown an increase in operating room cases and billing as well as an increase in surgeon satisfaction after the implementation of ACS. ${ }^{16}$

In addition to improved productivity, interest, and satisfaction, other studies have emphasized the ACS model as improving patient throughput. Multiple studies in North America, Australia and Asia, have demonstrated the efficiency and utility of an Acute Care Surgical model. The majority of these studies looked at the effectiveness of an ACS model as it pertained to appendectomies and cholecystectomies, with some also looking at small bowel obstructions, as these are the most common ACS operations performed. Studies demonstrated a statistically significant decrease in time to surgical consultation and time to the operating room, fewer complications, and a reduced length for appendectomies ${ }^{17-19}$ and cholecystectomies ${ }^{20}$ performed by surgeons in an ACS model.

\section{THE FUTURE OF ACS}

A recent survey of all graduates of approved AAST fellowship programs demonstrated that most graduates practice ACS in teaching hospitals designated at level I and level II trauma centers. Overwhelmingly, the fellows reported that the fellowship added value and prepared them well for practice $(C \mathrm{C}$ Burlew, unpublished data, 2016, used with permission). As interest in ACS as a specialty practice paradigm and as a training program grows, it is expected that more academic medical centers with mature ACS services will be applying for approved fellowship positions, at a rate of $\sim 2-3$ programs per year. The pace at which programs are embracing the training paradigm is accelerating, and may be related to the increasing number of fellowship-trained physicians working in these programs.

In April of 2016, the ABS released a statement regarding residency redesign that envisions a new paradigm for general surgery training, with the stated goal of greater autonomy and flexibility for the trainees. ${ }^{21}$ In this document, the ABS proposed a 'core plus' framework, which would consist of a core 4 years of general surgery training, followed by a variable number (1-3) of additional years of focused specialty training. In many ways, this is similar to the current $5+$ fellowship model widely embraced by roughly $80 \%$ of surgical trainees in the 


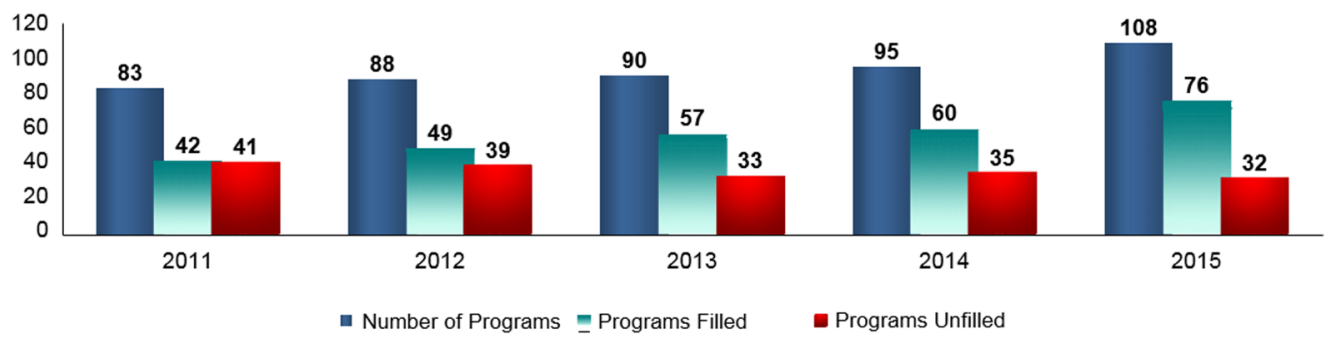

Positions

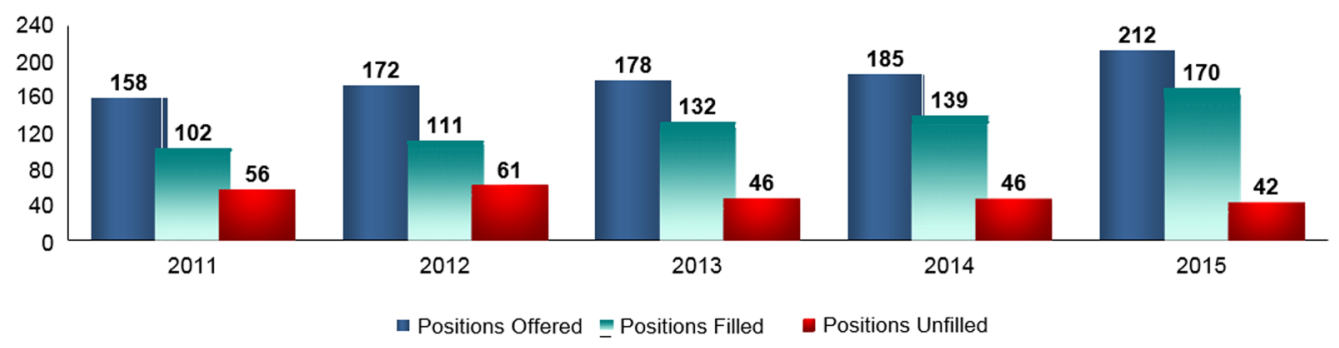

Applicants

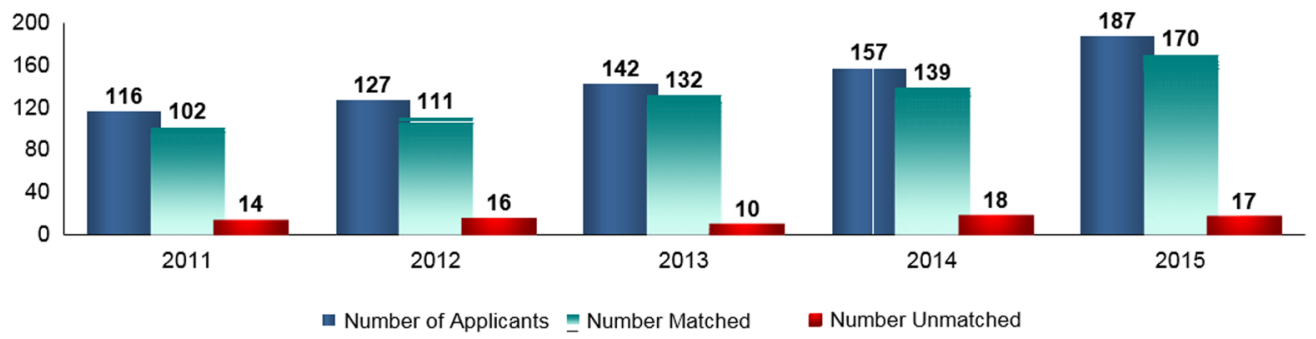

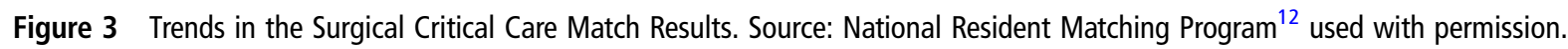

USA. The more unique feature of this proposal is that everyone would have to accomplish the 'plus' years of training, including an as yet undefined 'advanced general surgery fellowship'. Several unanswered questions persist regarding the statement released. Chief among these include how the ACGME and surgical Residency Review Committee (RRC) view the proposed changes, as all of general surgery training and 50\% of fellowship training currently fall under the egis of the ACGME and RRC, and not the ABS. Certainly not all programs could support all 'plus years', and some may not be convinced of the utility of this approach and may view it as the end of 'general surgery' in the USA. Further questions involve the future mechanisms for oversight of the many non-ACGME fellowships, such as ACS. Will they remain under the supervision of the specialty societies that supported their creation, such as the AAST, or will they be encompassed into a more formal ACGME-like training paradigm? Until these questions are more formally and completely addressed, the question of whether ACS training will become an ABS-certified specialty will remain unanswered. Current certification for those completing approved fellowships is provided by the AAST.

\section{SUMMARY}

The creation of ACS as a training and practice paradigm was driven by a declining interest in the field of trauma surgery, leading to the lack of surgeons available to take emergency calls and thereby resulting in poor access to emergency surgical care across the USA. Creation of ACS services allows the time-critical delivery of emergent and urgent surgical care despite an identified surgeon workforce shortage, bolstered by these specialty- trained surgeons in critical care, trauma and advanced emergency general surgery procedures. The field is once again attractive for its diversity of career options, and its tremendous benefits to society. Many of the initial concerns regarding the implementation of this model and introduction of this fellowship have not come to fruition. Specifically, resident education and non-trauma surgeons' operative logs have not been negatively impacted. However, our most critically ill surgical patients have benefited, with improved outcomes, more efficient care, and decreased mortality. Just as the needs of the injured patient drove the development of the field of trauma surgery, so did the needs of the emergency general surgery patient drive the development of the ACS paradigm. The training paradigm for the ACS fellows will continue to ensure that fully trained acute care surgeons are comfortable with a wide variety of anatomic exposures across all body regions. Acute care surgeons are uniquely positioned to impact healthcare cost containment and improve care in the USA as mandated by the Affordable Care Act of 2010. Cost savings can be actualized, and the system for care delivery optimized, by focusing on throughput and the use of standardized, evidence-based, consistent care. Acute care surgeons stand at the front line of care delivery for those patients who are most critically ill and for the injured surgical patients with time-sensitive diseases. Getting the right patient to the right venue at the right time is the paramount skill that the acute care surgeon, through training and experience, adds to the value equation. ${ }^{22}$

Competing interests None declared.

Provenance and peer review Commissioned; externally peer reviewed. 
Open Access This is an Open Access article distributed in accordance with the Creative Commons Attribution Non Commercial (CC BY-NC 4.0) license, which permits others to distribute, remix, adapt, build upon this work non-commercially, and license their derivative works on different terms, provided the original work is properly cited and the use is non-commercial. See: http://creativecommons.org/ licenses/by-nc/4.0/

\section{REFERENCES}

1 Division of Advocacy and Health Policy. A growing crisis in patient access to emergency surgical care. Bull Am Coll Surg 2006;91:8-19.

2 Committee on the Future of Emergency Care in the United States Health System. Hospital-Based Emergency Care: At the Breaking Point. Washington, DC: The National Academies Press, 2006. http://www.nationalacademies.org/hmd/Reports/ 2006/Hospital-Based-Emergency-Care-At-the-Breaking-Point.aspx

3 https://www.aamc.org/download/426260/data/physiciansupplyanddemand through2025keyfindings.pdf (accessed 2 Jul 2015).

4 IHS Inc. The complexities of physician supply and demand: projections from 2013 to 2025. Washington DC: Association of American Medical Colleges, 2015. https:// www.aamc.org/download/426242/data/ihsreportdownload.pdf (accessed $26 \mathrm{Mar}$ 2016).

5 Davis KA, Jurkovich GJ. An update on Acute Care Surgery: Emergence of Acute Care Surgery. ACS Surgery News 11 June 2015.

6 Dente CJ, Duane TM, Jurkovich GJ, et al. How much and what type: analysis of the first year of the acute care surgery operative case log. I Trauma Acute Care Surg 2014:76:329-39.

7 Davis KA, Dente CJ, Burlew CC, et al. Refining the operative curriculum of the acute care surgery fellowship. J Trauma Acute Care Surg 2015;78:192-6.

8 Shafi S, Aboutanos M, Brown CV, et al. Measuring anatomic severity of disease in emergency general surgery. J Trauma Acute Care Surg 2014;76:884-7.

9 Dinan KA, Davis JW, Wolfe MM, et al. An acute care surgery fellowship benefits a general surgical residency. J Trauma Acute Care Surg 2014;77:209-12.

10 Richardson JD, Miller FB. Will future surgeons be interested in trauma care? Results of a resident survey. J Trauma 1992;32:229-33; discussion 233-5.

11 Esposito TJ, Leon L, Jurkovich GJ. The shape of things to come: results from a national survey of trauma surgeons on issues concerning their future. J Trauma 2006;60:8-16
12 National Resident Matching Program. Results and data: 2015 main residency match $\circledast$. Washington DC: National Resident Matching Program, 2015. http://www. nrmp.org/wp-content/uploads/2015/02/Results-and-Data-SMS-2015.pdf (accessed 26 Mar 2016).

13 Coleman JJ, Esposito TJ, Rozycki GS, et al. Acute care surgery: now that we have built it, will they come? J Trauma Acute Care Surg 2013;74:463-9.

14 Wanis $\mathrm{KN}$, Hunter $\mathrm{AM}$, Harington $\mathrm{MB}$, et al. Impact of an acute care surgery service on timeliness of care and surgeon satisfaction at a Canadian academic hospital: a retrospective study. World J Emerg Surg 2014;9:4.

15 Barnes SL, Cooper CJ, Coughenour JP, et al. Impact of acute care surgery to departmental productivity. J Trauma 2011;71:1027-34.

16 Davis KA, Cabbad NC, Schuster KM, et al. Trauma team oversight improves efficiency of care and augments clinical and economic outcomes. J Trauma 2008;65:1236-42.

17 Cubas RF, Gómez NR, Rodriguez S, et al. Outcomes in the management of appendicitis and cholecystitis in the setting of a new acute care surgery service model: impact on timing and cost. J Am Coll Surg 2012;215:715-21.

18 Fu CY, Huang HC, Chen RJ, et al. Implementation of the acute care surgery model provides benefits in the surgical treatment of the acute appendicitis. Am J Surg 2014;208:794-9.

19 Pillai S, Hsee L, Pun A, et al. Comparison of appendicectomy outcomes: acute surgical versus traditional pathway. ANZ J Surg 2013;83:739-43.

20 Michailidou M, Kulvatunyou N, Friese RS, et al. Time and cost analysis of gallbladder surgery under the acute care surgery model. I Trauma Acute Care Surg 2014;76:710-13.

21 ABS statement Regarding Residency Redesign, 25 April 2016. http://www. absurgery.org/default.jsp?news_resredesign0416 (accessed 27 Apr 2016).

22 Frankel HL, Butler KL, Cuschieri J, et al. The role and value of surgical critical care, an essential component of Acute Care Surgery, in the Affordable Care Act: a report from the Critical Care Committee and Board of Managers of the American Association for the Surgery of Trauma. J Trauma Acute Care Surg 2012;73:20-6.

23 Dill MJ, Salsberg ES. The Complexities of Physician Supply and Demand: Projections Through 2025. Washington, DC. Association of American Medical Colleges, 2008 https://members.aamc.org/eweb/upload/The Complexities of Physician Supply.pdf

24 The American Associationfor Surgery of Trauma. List of AAST Approved Programs \& Information. TraumaSource. http://www.aast.org/list-of-aast-approved-programs (accessed 4 May 2016). 